Among the other organisms isolated 29 patients had Haemophilus influenzae (Reiff Mean 3.7, SD 3.5). This mean Reiff score was significantly lower than for $P$. aeruginosa, $(\mathrm{p}=0.03)$ - figure 1 .

Streptococcus pneumoniae (Reiff Mean 3.4), Moraxella catarrhalis (Reiff Mean 3.7) and Staphylococcus aureus (Reiff Mean 3.2), were also isolated in lesser numbers and a further 28 patients had a mix of other colonising organisms (Reiff Mean 3.5).

Conclusion More severe radiological bronchiectasis is associated with the presence of bacterial colonisation and particularly colonisation with Pseudomonas aeruginosa. This simple assessment of radiological severity may be a useful clinical tool in non-CF bronchiectasis.

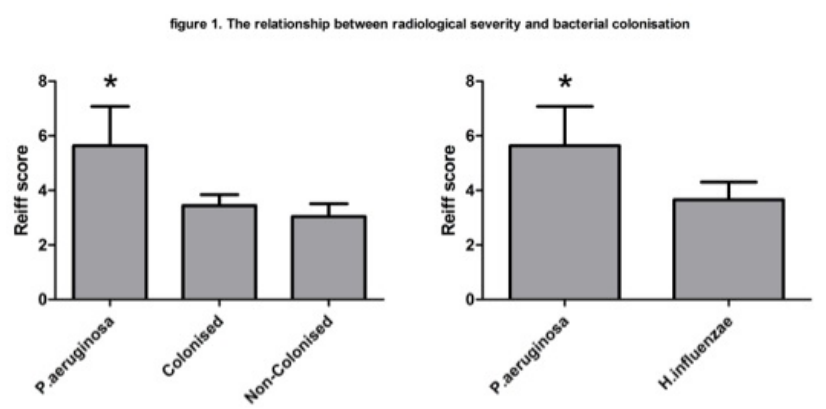

Abstract P174 Figure 1 Radiological severity and bacterial colonisation

\section{P175 THE SHORT TERM VARIABILITY OF SPUTUM MICROBIOLOGY IN NON-CF BRONCHIECTASIS}

doi:10.1136/thoraxjnl-2012-202678.236

MC Coleman, C Hennessy, D Bilton, R Wilson, MR Loebinger. Royal Brompton Hospital, London, UK

Introduction It is recommended that non-CF bronchiectasis patients have sputum cultured annually. This prospective, cohort study is the first to collect monthly cultures to assess this practise by recording the short term variability of sputum bacteriology.

Method 85 patients with non-CF bronchiectasis and daily sputum production were recruited between December 2010 and May 2011. Patients completed daily symptom diaries, and spirometry and sputum samples were collected monthly for 6 months.

Results 58/85 were female, average age 58 (range 17-82). Most common aetiologies were idiopathic $41 / 85$ and post infective 19/85. 64/85 completed follow up.

There were 417 sputum cultures of which only 265 cultured an organism (incl. 130 Pseudomonas aeruginosa (PA), 37 Staphlococcus aureus, 34 Haemophilus influenzae.) 10/64 patients grew no organism throughout the study despite monthly samples. 30/64 had one or more positive culture for PA, including 6 patients with first isolates. Of those with positive cultures, 28/64 patients grew the same organism in all positive cultures (15/64 grew PA only) whereas $26 / 64$ had variability in the microbe isolated. A total of 37 exacerbations occurred at time of clinic visit. Of these 4/37 were associated with growth of a pathogen not previously isolated in this patient. However, 20/37 sputum samples at the time of clinical exacerbation showed no growth despite no prior antibiotic use. 22 patients used prophylactic antibiotics of whom 14 grew PA and 2 grew no organism.

122 infective exacerbations resulted in the use of 145 antibiotics courses. 14/64 had no exacerbations over 6 months. 9/64 had one and 41/64 had two or more (median 2). Spirometry and symptom scores remained relatively stable throughout the 6 months and were not significantly different in patients with $\geq 2$ exacerbations.

Conclusion This study demonstrates the limitations of annual sputum cultures. There was significant microbe variability and importantly a significant number of first PA isolations over a short follow-up period. Furthermore, a large percentage of patients had no microbe isolated (including at exacerbation) suggesting a possible use of future molecular microbe techniques.

\section{P176 COPD-RELATED BRONCHIECTASIS; A REAL CLINICAL ENTITY WITH IMPACT ON DISEASE COURSE AND OUTCOMES}

doi:10.1136/thoraxjnl-2012-202678.237

'EH Baker, ${ }^{1} \mathrm{~N}$ Kumar, ${ }^{2} \mathrm{D}$ Lai, ${ }^{1} \mathrm{~B}$ Sansom, ${ }^{1} \mathrm{~A}$ Nair, ${ }^{1} \mathrm{~V}$ Vlahos. ${ }^{1}$ St George's University of London, London, UK; ${ }^{2}$ Chelsea and Westminster Hospital, London, UK

Introduction and Objectives Non-CF bronchiectasis is defined as 'symptoms of persistent or recurrent bronchial sepsis related to irreversibly damaged and dilated bronchi' [BTS guidelines 2010]. Radiographic evidence of 'damaged and dilated bronchi' can be seen on CT Thorax in up to $50 \%$ of COPD patients. However the contribution of radiographic bronchiectasis to the clinical course of COPD is not fully understood. We aimed to determine the impact of bronchiectasis on lung function, sputum microbiology and outcomes in COPD patients, independent of coexisting emphysema and bronchial wall thickening (BWT).

Methods COPD patients admitted with first exacerbation 1998-2008 were identified retrospectively using ICD10 codes J44.0,1,8,9. Patients with high resolution CT images within 2 years of admission were included. CT scans were graded by consensus of 2 senior thoracic radiologists for severity of bronchiectasis, emphysema and BWT on a 5 point scale (0-absent, 1-minor, 2-mild, 3-moderate, 4-severe). Operational definitions were set prior to scan review and radiologists were blinded to clinical parameters.

Results 406 patients ( $71 \pm 11$ years, $56 \%$ male, $\mathrm{FEV}_{1} 52 \pm 23 \%$ predicted) were included. 278 (69\%) patients had bronchiectasis: minor, 112 (40\%); mild, 81 (29\%); moderate, 62 (22\%); severe 23 (8\%). There was considerable overlap between bronchiectasis and other pathologies (figure). Bronchiectasis severity correlated with severity of BWT $(r=0.276, p<0.001)$ and emphysema $(r=0.120$, $p=0.015$ ). After adjustment for severity of emphysema and BWT, increasing severity bronchiectasis was not an independent predictor of lung function parameters, but independently determined isolation of Pseudomonas aeruginosa (Odds ratio (OR) 1.39 (95\% CI 1.07$1.80), p=0.013)$ and atypical mycobacteria from sputum cultures (OR 2.44 (95\% CI 1.04-5.69), p=0.040). After correction for increasing severity emphysema, BWT, age, gender and comorbidities, increasing severity bronchiectasis determined annual admissions (regression coefficient $\mathrm{B}=0.14$ (95\% CI $0.00-0.28), \mathrm{p}=0.044$ ) and inpatient days $(B=2.1$ (95\% CI $0.8-3.4), p=0.001)$ for respiratory causes, but did not influence survival from first hospital admission $(p=0.257)$.

Conclusions Radiographic bronchiectasis in COPD patients is associated with increased respiratory infection and hospitalisation, independent of coexisting emphysema and BWT. COPD-related bronchiectasis is therefore a diagnosis with important clinical implications. Further research should determine whether treatment strategies for non-CF bronchiectasis can improve the clinical course of COPD-related bronchiectasis.

\section{P177 OUTCOMES OF PSEUDOMONAS AERUGINOSA (PA) ERADICATION IN NON-CYSTIC FIBROSIS BRONCHIECTASIS. FORCED VITAL CAPACITY (FVC) AND LATENT PERIOD FROM GROWTH TO ERADICATION ARE SIGNIFICANT VARIABLES IN ERADICATION SUCCESS}

doi:10.1136/thoraxjnl-2012-202678.238

S Ejiofor, G Packer, K Mckinley, J Whitehouse. Birmingham Heartlands Hospital-Heart of England NHS Trust, Birmingham, England 
Figure. Overlap between increasing severity bronchiectasis and increasing severity: a) bronchial wall thickening; b) emphysema

a) Bronchial wall thickening

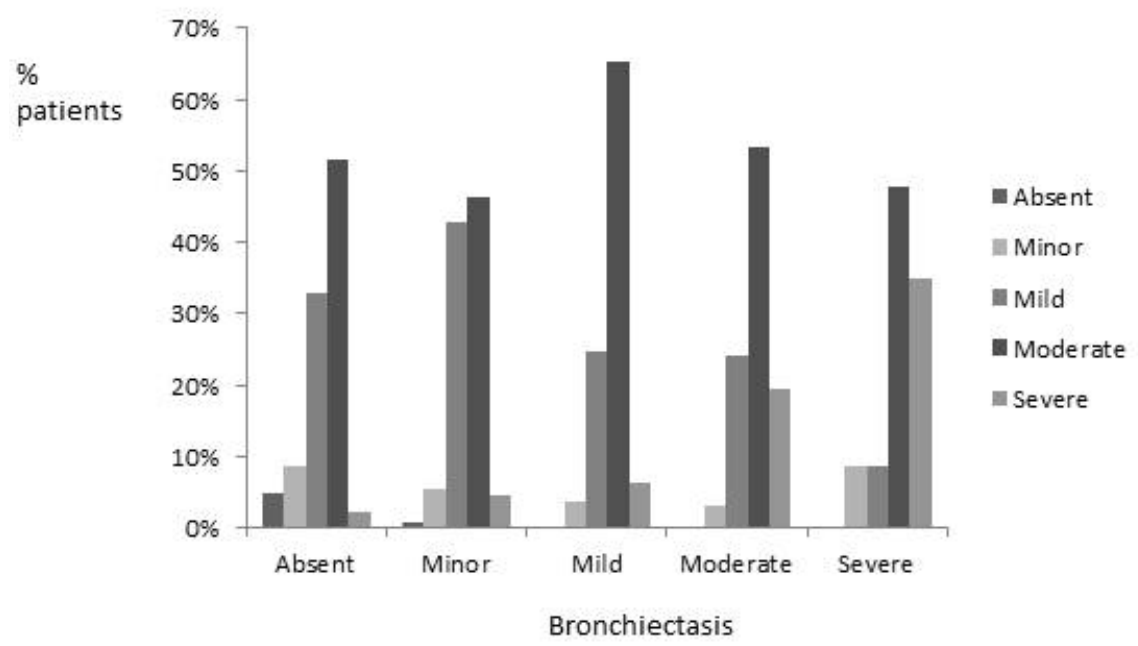

b) Emphysema

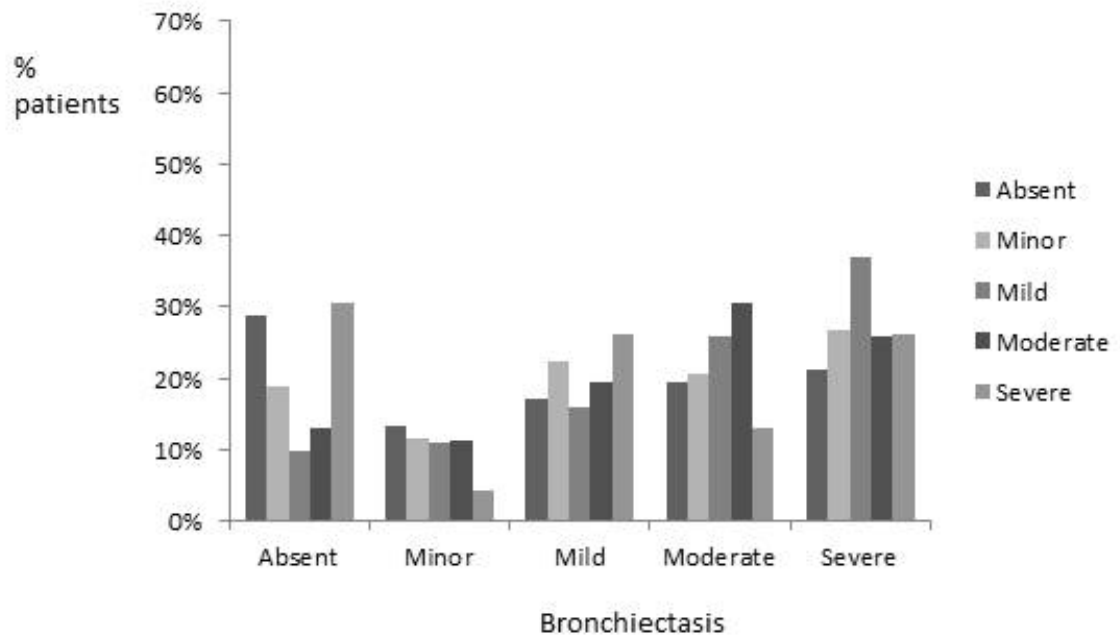

Abstract P176 Figure 1

Introduction PA colonisation is associated with a more rapid decline in lung function and poorer health outcomes and possibly increased mortality in non cystic fibrosis bronchiectasis. ${ }^{1,2}$ Early aggressive antibiotic therapy is aimed at negating these deleterious effects and is recommended by the British Thoracic Society (BTS). ${ }^{3}$ Within Heart of England NHS Trust a policy of PA eradication has existed since 2005.

Method All patients with a clinical presentation of and HRCTconfirmed bronchiectasis were identified retrospectively from a respiratory outpatient database created in 2005. Patients were included in the study if they a) cultured PA in sputum (usually 1st growth) b) underwent treatment with eradication intent and c) had at least 12 months follow up from the initiation of eradication therapy. Eradication was defined as three negative sputum samples taken following eradication treatment. Eradication regimens broadly followed the BTS guidance. Mann U Whitney and $\chi 2$ tests were used in statistical analyses.

\section{Results see table1}

Eradication of PA was achieved in $51 / 72$ (71\%) of patients. Of those eradicated $36 / 51$ (71\%) did not reculture PA to date. Mean time in months from first growth to eradication (6.82 vs 16.19) was significantly lower in the group successfully eradicated $(p<0.0001)$. FVC \% predicted was significantly higher in the PA eradicated group $(p=0.006)$.

Conclusions Eradication of PA can be undertaken with relatively high success rates and with almost three quarters remaining free from PA. Good lung function and a short latent time from growth to eradication are independently associated with eradication success.

\section{References}

1. Martínez-García M A et al. Chest 2007; 132:1565-1572.

2. Loebinger MR et al. Eur Respir J 2009 34(4) 843-849.

3. BTS guideline for non CF bronchiectaisis Thorax July 2010 65 (1). 
Abstract P177 Table 1

\begin{tabular}{llll}
\hline${ }^{*}$ Age (years) & & 64.1 SD 12.8 & \\
Male:Female & & $51: 21$ & \\
${ }^{*} \mathrm{FEV}_{1} \%$ predicted & $\mathrm{FEV}_{1}$ (L) & $58 \%(\mathrm{SD} 23 \%)$ & 1.32 (SD 0.66) \\
${ }^{*} \mathrm{FVC} \%$ predicted & $\mathrm{FVC} \mathrm{(L)}$ & $79 \%(S D 23 \%)$ & 2.23 (SD 0.89) \\
\hline${ }^{*}$ mean values & &
\end{tabular}

\section{P178 INFORMATION AND EDUCATION NEEDS OF PATIENTS WITH BRONCHIECTASIS: A QUALITATIVE INVESTIGATION}

doi:10.1136/thoraxjnl-2012-202678.239

${ }^{1} \mathrm{KL}$ M Hester, ${ }^{1} \mathrm{~A}$ De Soyza, ${ }^{2} \mathrm{~T}$ Rapley. ${ }^{1}$ Freeman Hospital, Newcastle upon Tyne, UK; ${ }^{2}$ Newcastle University, Newcastle upon Tyne, UK

Introduction Bronchiectasis is a chronic lung condition, characterised by dilated bronchi, causing breathlessness and chronic productive cough, with intermittent infective exacerbations. Patients often have recurrent, costly hospital admissions, a poorer quality of life, and clinically significant fatigue. ${ }^{1}$ Current estimates suggest there are 26,000 adults with the condition in the UK. There is little patient information currently available. Patients reported in a questionnaire that information improved confidence. ${ }^{2}$ Information and education could improve understanding and empower patients to self-manage. Timely recognition and appropriate management of exacerbations could improve disease stability. Qualitative investigation of patients' needs will focus plans for resource development, ensure a user-driven format and content, and improve patient engagement and benefit.

Aims To identify, explore and understand the information needs within this patient group, describing the issues in detail.

Methods An exploratory qualitative study was conducted. Eight patients ( 6 female, 2 male, median age 66 years, range 33-67) were recruited from an adult bronchiectasis clinic in the North of England. In-depth semi-structured interviews were conducted, audio-recorded and transcribed. Thematic analysis was undertaken to identify common themes.

Results Four main interlinking themes emerged:

1. Information: patients felt available information was lacking and accessible resources were needed. A crucial factor for acceptance was trust, with participants expressing a need for information to originate from a reputable source. The majority preferred a web-based resource.

2. Medical care and support: Patients felt that a specialist clinic provided support, help and information, yet there was a need for accessible information outside this setting.

3. Diagnosis experience: this had a big impact on patients; often little information and support was given. They felt more information at an early stage would help patients to cope with and manage bronchiectasis.

4. Living with bronchiectasis and its impact: information and knowledge seemed to be fundamental to patients learning to live with and manage their condition.

Conclusions These findings suggest patients want more information. With trustworthy resources patients could be better equipped to understand and manage bronchiectasis. Further work is planned to inform development and implementation of a user-driven information resource for patients with bronchiectasis.

\section{References}

1. Hester KLM, Macfarlane JG, Tedd H, Jary H, McAlinden P, Rostron L, Small T, Newton JL, De Soyza A. Fatigue in bronchiectasis. OJM 2012 Mar; 105(3):235-40.

2. Hester KLM, McAlinden P, De Soyza A, Education and information in bronchiectasis: what do patients want? European Respiratory Journal. 2011; 38 supplement 55: P3622.

\section{P179 NEBULISED 7\% HYPERTONIC SALINE AS AN ADJUNCT TO AIRWAY CLEARANCE CAN BE COMMENCED SAFELY IN A SUPERVISED ENVIRONMENT WITHOUT THE NEED FOR A FORMAL BRONCHOCONSTRICTION TRIAL}

doi:10.1136/thoraxjnl-2012-202678.240

${ }^{1} \mathrm{NG}$ Mills, ${ }^{1} \mathrm{JR}$ Willis, ${ }^{2} \mathrm{SP}$ Range, ${ }^{2} \mathrm{CM}$ Ohri. 'Physiotherapy Department, Glenfield Hospital, Leicester, UK; 'Institute for Lung Health, Glenfield Hospital, Leicester, UK

Background Nebulised Hypertonic Saline (HTS) has been shown to increase water content of airway surface liquid, enhance mucociliary clearance, reduce exacerbation frequency and improve quality of life in Cystic Fibrosis (CF) and non-CF Bronchiectasis. Historically HTS was associated with bronchoconstriction, therefore formal first dose bronchoconstriction trials (BCT) were performed. Recent studies have shown better tolerance that improves over up to 10 doses (Elkins 2006). We have observed that a fall in FEV1 $>15 \%$ following nebulised HTS rarely correlates with adverse signs or symptoms, questioning the need for a BCT. This study aimed to assess our use of HTS, its safety and tolerance without BCT, and its positive effects.

Methods A senior physiotherapist completed a proforma for all patients commencing HTS over 6 months. HTS was used for different respiratory conditions to aid mucociliary clearance. All patients were pre-dosed with a bronchodilator, and had a closely supervised $1^{\text {st }}$ test dose, replacing formal BCT.

Results 54 patients aged 19-87 years (mean 54, median 61) commenced 7\% HTS. 35 were inpatients and 19 outpatients. The primary diagnoses were: non-CF Bronchiectasis (20), post thoracic surgery (17), CF (13), Pneumonia (2), long term tracheostomy (1), and COPD with Laryngectomy (1). All patients experienced positive effects, listed in table 1 . HTS prescription was individualised: BD regime (42), TDS (8), ODS (4). 13 patients (24\%) experienced adverse effects - increased cough (13), slight chest tightness (3), a $5 \%$ decrease in oxygen saturations (1). All 13 patients and physiotherapists considered the adverse effects sufficiently mild to continue HTS and they subsequently reduced significantly after up to 5 doses. If no adverse effects were experienced during the test dose, no further adverse effects occurred with continued use.

Conclusion This observational study demonstrates that HTS has positive effects in a wide variety of respiratory conditions. We have replaced BCT with a supervised test dose as it was found to a be a safe and efficient evaluation. Over 6 months HTS was well tolerated, with only mild adverse effects, which reduced over subsequent doses. Importantly, there were no adverse effects in patients who had previously undergone an uneventful test dose.

\section{Abstract P179 Table 1}

\begin{tabular}{lc}
\hline Positive effects of test dose & Number \\
\hline$\uparrow$ Sputum cleared & 32 \\
$\uparrow$ Sputum cleared with $\uparrow$ ease & 21 \\
$\downarrow$ Sputum retention & 3 \\
$\uparrow$ Sp02 & 5 \\
$\uparrow$ Lung volume on CXR & 3 \\
$\downarrow$ suction required & 2 \\
Chest felt clearer between treatments & 2 \\
$\downarrow$ Viscosity sputum & 1 \\
$\downarrow$ Work of breathing & 1 \\
$\downarrow$ Frequency of physiotherapy needed & 1 \\
$\downarrow$ Anxiety with airway clearance & 1 \\
$\uparrow$ Clearance nasal secretions & 1 \\
Facilitated weaning from the ventilator & 1 \\
\hline
\end{tabular}

